\title{
YEH CONVOLUTION OF WHITE NOISE FUNCTIONALS ${ }^{\dagger}$
}

\author{
UN CIG JI*, YOUNG YI KIM AND YOON JUNG PARK
}

\begin{abstract}
In this paper, we study the Yeh convolution of white noise functionals. We first introduce the notion of Yeh convolution of test white noise functionals and prove a dual property of the Yeh convolution. By applying the dual object of the Yeh convolution, we study the Yeh convolution of generalized white noise functionals, which is a non-trivial extension. Finally, we study relations between the Yeh convolution and Fourier-Gauss, Fourier-Mehler transform.
\end{abstract}

AMS Mathematics Subject Classification : 60H40, 46F25.

Key words and phrases : white noise theory, Yeh convolution, FourierGauss transform, Fourier-Mehler transform.

\section{Introduction}

Since Yeh in [14] introduced the convolution $\phi * \psi$, called the Yeh convolution, of Wiener functionals $\phi$ and $\psi$ by

$$
\phi * \psi(y) \equiv \int_{C_{0}[0,1]} \phi\left(\frac{1}{\sqrt{2}} x+\frac{1}{\sqrt{2}} y\right) \psi\left(\frac{1}{\sqrt{2}} x-\frac{1}{\sqrt{2}} y\right) m(d x)
$$

for $y \in C_{0}[0,1]$, whenever the integral exists, where $\left(C_{0}[0,1], m\right)$ is the (standard) Wiener space with the Wiener measure $m$, several authors studied the Yeh convolution with relations between first variations and various transforms (see [5] and its references).

On the other hand, the white noise theory initiated by Hida [4] to give rigorous meaning of white noise as the time derivative of the Brownian motion has been extensively developed with wide applications to stochastic calculus, mathematical finance and mathematical physics, etc. The convolution product by Kuo [8] and Fourier-Gauss transforms [2, 8] of white noise functionals are important

Received March 19, 2013. Revised April 27, 2013. Accepted April 30, 2013. ${ }^{*}$ Corresponding author. †This work was supported by the research grant of the Chungbuk National University in 2011.

(c) 2013 Korean SIGCAM and KSCAM. 
applications to infinite dimensional (harmonic) analysis. Also, the convolution products of white noise operators were studied in [6].

In this paper, we study the Yeh convolution of white noise functionals. Since the test white noise functionals have nice regular properties, the Yeh convolution of test white noise functionals is well defined (see [5]). However, from singular properties of the generalized white noise functionals, the extension of Yeh convolution to the generalized white noise functionals is not trivial. Hence main purpose of this paper is to develop a method to give a rigorous meaning of the Yeh convolution of generalized white noise functionals. For our purpose, we study a dual property (see Theorem 3.4) of the Yeh convolution of test white noise functionals and then as an application of the dual object, we study the Yeh convolution of generalized white noise functionals. We also study relations between the Yeh convolution and Fourier-Gauss, Fourier-Mehler transform (see Theorems 3.3 and 4.4).

This paper is organized as follows: In Section 2 we recall basic notions and well-known results in white noise theory, which are necessary for our study, see $[12,8]$. In Section 3 we introduce the notion of Yeh convolution of test white noise functionals and prove a dual property of the Yeh convolution. We study a relations between the Yeh convolution and Fourier-Gauss transform. In Section 4 we study the extension of the Yeh convolution to the convolution of generalized white noise functionals. Finally, we study a relation between the Yeh convolution and Fourier-Mehler transform.

\section{Preliminaries}

Let $H_{\mathbb{R}}$ be a real separable Hilbert space with inner product $\langle\cdot, \cdot\rangle$ and let $A$ be a positive selfadjoint operator on $H_{\mathbb{R}}$ satisfying that there exists an orthonormal basis $\left\{e_{n}\right\}_{n=1}^{\infty}$ and an increasing sequence $\left\{l_{n}\right\}_{n=1}^{\infty}$ with $l_{1}>1$ and $\sum_{n=1}^{\infty} l_{n}^{-2}<$ $\infty$ such that

$$
A e_{n}=l_{n} e_{n}, \quad n=1,2, \cdots
$$

We note that $\rho:=\left\|A^{-1}\right\|_{\mathrm{OP}}=l_{1}^{-1}<1$ and $\left\|A^{-1}\right\|_{\mathrm{HS}}^{2}<\infty$. Then by the standard construction from $H_{\mathbb{R}}$ and $A$ (see $[8,12]$ ), we have a Gelfand triple:

$$
E_{\mathbb{R}} \subset H_{\mathbb{R}} \subset E_{\mathbb{R}}^{*},
$$

where $E_{\mathbb{R}}^{*}$ is the strong dual space of $E_{\mathbb{R}}$. In fact, the topology of $E_{\mathbb{R}}$ is defined by the Hilbertian norms $\left\{|\cdot|_{p} \equiv\left|A^{p} \cdot\right|\right\}_{p \geq 0}$, where $|\cdot|$ is the norm generated by $\langle\cdot, \cdot\rangle$ and then $E_{\mathbb{R}}$ becomes a countable Hilbert nuclear space. By taking complexificaion of (2) we have the complex Gelfand triple:

$$
E \subset H \subset E^{*},
$$

where $E=E_{\mathbb{R}}+i E_{\mathbb{R}}$ and $H=H_{\mathbb{R}}+i H_{\mathbb{R}}$. The canonical bilinear form on $E^{*} \times E$ is denoted by $\langle\cdot, \cdot\rangle$ again. 
The (Boson) Fock space $\Gamma(H)$ over $H$ is defined by

$$
\Gamma(H)=\left\{\phi=\left(f_{n}\right)_{n=0}^{\infty}: f_{n} \in H^{\widehat{\otimes} n}, \quad\|\phi\|^{2}=\sum_{n=0}^{\infty} n !\left|f_{n}\right|_{0}^{2}<\infty\right\},
$$

where $H^{\widehat{\otimes} n}$ is the $n$-fold symmetric tensor product of $H$ and $H^{\widehat{\otimes} 0}=\mathbb{C}$. Let $\Gamma(A)$ be the second quantization of the operator $A$ defined by

$$
\Gamma(A) \phi=\left(A^{\otimes n} f_{n}\right)_{n=0}^{\infty}, \quad \phi=\left(f_{n}\right)_{n=0}^{\infty} \in \Gamma(H),
$$

and then $\Gamma(A)$ is a positive selfadjoint operator with $\left\|\Gamma(A)^{-1}\right\|_{\mathrm{OP}}<1$ and $\left\|\Gamma(A)^{-1}\right\|_{\mathrm{HS}}^{2}<\infty$. By the standard construction from $\Gamma(H)$ and $\Gamma(A)$, we have a Gelfand triple:

$$
(E) \subset \Gamma(H) \subset(E)^{*} .
$$

In fact, the (projective) topology of $(E)$ is determined by the family $\left\{\|\cdot\|_{p}\right\}_{p \geq 0}$ of norms defined by

$$
\|\phi\|_{p}^{2}=\sum_{n=0}^{\infty} n !\left|f_{n}\right|_{p}^{2}, \quad \phi=\left(f_{n}\right)_{n=0}^{\infty} \in(E) .
$$

It is known that for each $\Phi \in(E)^{*}$ there exists a unique sequence $\left(F_{n}\right)_{n=0}^{\infty}$ with $F_{n} \in\left(E^{\otimes n}\right)_{\mathrm{sym}}^{*}$ such that

$$
\langle\langle\Phi, \phi\rangle\rangle=\sum_{n=0}^{\infty} n !\left\langle F_{n}, f_{n}\right\rangle, \quad \phi=\left(f_{n}\right)_{n=0}^{\infty} \in(E),
$$

in this case, $\Phi$ is denoted by $\Phi=\left(F_{n}\right)_{n=0}^{\infty}$.

It follows from the Bochner-Minlos theorem that there exists a unique probability measure $\mu$ on $E_{\mathbb{R}}^{*}$ such that its characteristic function is given by

$$
\exp \left(-\frac{1}{2}|\xi|_{0}^{2}\right)=\int_{E_{\mathbb{R}}^{*}} e^{i\langle x, \xi\rangle} \mu(d x), \quad \xi \in E_{\mathbb{R}} .
$$

The above probability measure $\mu$ is called the standard Gaussian measure on $E_{\mathbb{R}}^{*}$ and the probability space $\left(E_{\mathbb{R}}^{*}, \mu\right)$ is referred to as the (standard) Gaussian space. The unitary isomorphism between $L^{2}\left(E_{\mathbb{R}}^{*}, \mu ; \mathbb{C}\right)$ and $\Gamma(H)$, called the Wiener-Itô-Segal isomorphism, is uniquely determined by the correspondence:

$$
\begin{aligned}
\Gamma(H) \ni \phi_{\xi} & =\left(1, \xi, \frac{\xi^{\otimes 2}}{2 !}, \ldots, \frac{\xi^{\otimes n}}{n !}, \ldots\right) \\
& \longleftrightarrow \phi_{\xi}(x)=e^{\langle x, \xi\rangle-\frac{1}{2}\langle\xi, \xi\rangle} \in L^{2}\left(E_{\mathbb{R}}^{*}, \mu ; \mathbb{C}\right),
\end{aligned}
$$

where $\phi_{\xi}$ is called an exponential vector (or coherent state) and $\phi_{\xi}(x)$ is called the Gaussianization of $\phi_{\xi}$.

Since $\left\{\phi_{\xi_{1}} \otimes \cdots \otimes \phi_{\xi_{m}}: \xi_{i} \in E, i=1,2, \ldots, m\right\}$ spans a dense subspace of $(E)^{\otimes m}$, every $\Xi \in \mathcal{L}\left((E)^{\otimes m},\left((E)^{\otimes n}\right)^{*}\right)$ is uniquely determined by the function $G: E^{m+n} \rightarrow \mathbb{C}$ defined by

$$
G\left(\xi_{1}, \ldots, \xi_{m}, \eta_{1}, \ldots, \eta_{n}\right)=\left\langle\left\langle\Xi\left(\phi_{\xi_{1}} \otimes \cdots \otimes \phi_{\xi_{m}}\right), \phi_{\eta_{1}} \otimes \cdots \otimes \phi_{\eta_{n}}\right\rangle\right\rangle
$$


for $\xi_{1}, \ldots, \xi_{m}, \eta_{1}, \ldots, \eta_{n} \in E$. In particular, for $\Xi \in \mathcal{L}\left((E),(E)^{*}\right)$, the form given as in (3) is denoted by $\widehat{\Xi}$ and called the symbol of $\Xi$.

Theorem 2.1 ([7]). A Gâteaux-entire function $G: E^{\otimes m+n} \rightarrow \mathbb{C}$ is expressed in the form (3) with $\Xi \in \mathcal{L}\left((E)^{\otimes m},\left((E)^{\otimes n}\right)^{*}\right)$ if and only if there exist constant numbers $C \geq 0, K \geq 0$ and $p \geq 0$ such that

$$
\left|G\left(\xi_{1}, \ldots, \xi_{m}, \eta_{1}, \ldots, \eta_{n}\right)\right|^{2} \leq C e^{K\left(\sum_{j=1}^{m}\left|\xi_{j}\right|_{p}^{2}+\sum_{k=1}^{n}\left|\eta_{k}\right|_{p}^{2}\right)}
$$

for any $\xi_{1}, \ldots, \xi_{m}, \eta_{1}, \ldots, \eta_{n} \in E$. Moreover $\Xi \in \mathcal{L}\left((E)^{\otimes m},(E)^{\otimes n}\right)$ if and only if for any $\epsilon>0$ and $p \geq 0$ there exist constant numbers $C \geq 0$ and $q \geq 0$ such that

$$
\left|G\left(\xi_{1}, \ldots, \xi_{m}, \eta_{1}, \ldots, \eta_{n}\right)\right|^{2} \leq C e^{\epsilon\left(\sum_{j=1}^{m}\left|\xi_{j}\right|_{p+q}^{2}+\sum_{k=1}^{n}\left|\eta_{k}\right|_{-p}^{2}\right)}
$$

for any $\xi_{1}, \ldots, \xi_{m}, \eta_{1}, \ldots, \eta_{n} \in E$.

For more study of analytic characterization theorems in white noise theory, we refer to $[13,9,11,1]$.

For each $\kappa_{l, m} \in\left(E^{\otimes l+m}\right)^{*}$, by applying Theorem 2.1 we can see that there exists an operator $\Xi_{l, m}\left(\kappa_{l, m}\right) \in \mathcal{L}\left((E),(E)^{*}\right)$, called an integral kernel operator, such that

$$
\widehat{\Xi}(\xi, \eta)=\left\langle\kappa_{l, m}, \eta^{\otimes l} \otimes \xi^{\otimes m}\right\rangle e^{\langle\xi, \eta\rangle}, \quad \xi, \eta \in E .
$$

Note that $\Xi_{l, m}\left(\kappa_{l, m}\right) \in \mathcal{L}((E),(E))$ if and only if $\kappa_{l, m} \in E^{\otimes l} \otimes\left(E^{\otimes m}\right)^{*}$. For $f \in E^{*}$, we write

$$
\Xi_{0,1}(f)=a(f), \quad \Xi_{1,0}(f)=a^{*}(f) .
$$

The operator $a(f)$ and $a^{*}(f)$ are called the annihilation and creation operator, respectively.

Let $\tau$ be the trace corresponding to the identity operator $I \in \mathcal{L}(E, E)$ under the canonical isomorphism $\mathcal{L}\left(E, E^{*}\right) \cong(E \otimes E)^{*}$ by the kernel theorem, that is,

$$
\langle\tau, \eta \otimes \xi\rangle=\langle\xi, \eta\rangle, \quad \xi, \eta \in E .
$$

The Gross Laplacian [3] $\Delta_{\mathrm{G}} \in \mathcal{L}((E),(E))$ is defined by $\Delta_{\mathrm{G}}=\Xi_{0,2}(\tau)$ and then we have

$$
\widehat{\Delta_{\mathrm{G}}}(\xi, \eta)=\langle\xi, \xi\rangle e^{\langle\xi, \eta\rangle}, \quad \xi, \eta \in E .
$$

\section{Convolutions of Test White Noise Functionals}

In this section, we study the Yeh convolution of test white noise functionals.

3.1. Translation and Dilation Operators. For each $y \in E_{\mathbb{R}}^{*}$, the translation operator $\mathcal{T}_{y}$ on $(E)$ is defined by

$$
\mathcal{T}_{y} \phi(x)=\phi(x+y), \quad x \in E_{\mathbb{R}}^{*} .
$$

In fact, for $\xi \in E$ we have

$$
\mathcal{T}_{y} \phi_{\xi}=e^{\langle y, \xi\rangle} \phi_{\xi}=e^{a(y)} \phi_{\xi}, \quad \widehat{\mathcal{T}_{y}}(\xi, \eta)=e^{\langle y, \xi\rangle+\langle\xi, \eta\rangle} .
$$

Therefore, by applying Theorem 2.1, we can see that $\mathcal{T}_{y} \in \mathcal{L}((E),(E))$. 
For each $\alpha \in \mathbb{C}$, by applying Theorem 2.1 we can easily see that there exists an operator $\mathcal{D}_{\alpha} \in \mathcal{L}((E),(E))$ such that

$$
\widehat{\mathcal{D}_{\alpha}}(\xi, \eta)=e^{\alpha\langle\xi, \eta\rangle+\frac{\alpha^{2}-1}{2}\langle\xi, \xi\rangle}, \quad \xi, \eta \in E
$$

which implies that

$$
\mathcal{D}_{\alpha} \phi_{\xi}(x)=e^{\frac{\alpha^{2}-1}{2}\langle\xi, \xi\rangle} \phi_{\alpha \xi}(x)=\phi_{\xi}(\alpha x), \quad x \in E_{\mathbb{R}}^{*}, \quad \xi \in E .
$$

Since the exponential vectors $\phi_{\xi}$ span a dense subspace of $(E)$ and $\mathcal{D}_{\alpha}$ is continuous,

$$
\mathcal{D}_{\alpha} \phi(x)=\phi(\alpha x), \quad x \in E_{\mathbb{R}}^{*}, \quad \phi \in(E) .
$$

Therefore, $\mathcal{D}_{\alpha}$ is called the dilation. Moreover, we have

$$
\mathcal{D}_{\alpha}=\Gamma(\alpha I) e^{\frac{1}{2} \Delta_{\mathrm{G}}\left(\left(\alpha^{2}-1\right) I\right)} .
$$

For each $\alpha \in \mathbb{C}$ and $y \in E^{*}$, put

$$
\mathcal{R}_{\alpha, y}=\mathcal{D}_{\alpha} \mathcal{T}_{y} .
$$

Then for any $\xi \in E$, we have

$$
\mathcal{R}_{\alpha, y} \phi_{\xi}(x)=\phi_{\xi}(\alpha x+y)=\phi_{\alpha \xi}(x) \phi_{\xi}(y) e^{\frac{\alpha^{2}}{2}\langle\xi, \xi\rangle},
$$

which implies that

$$
\widehat{\mathcal{R}_{\alpha, y}}(\xi, \eta)=e^{\alpha\langle\xi, \eta\rangle+\frac{\alpha^{2}-1}{2}\langle\xi, \xi\rangle+\langle y, \xi\rangle}, \quad \xi, \eta \in E .
$$

Thus we have the following expression:

$$
\mathcal{R}_{\alpha, y}=\Gamma(\alpha I) e^{\frac{1}{2} \Delta_{\mathrm{G}}\left(\left(\alpha^{2}-1\right) I\right)} e^{a(y)} \in \mathcal{L}((E),(E)) .
$$

3.2. Convolutions of Test White Noise Functionals. We start with the following lemma for the existence of the operator $C^{l}$.

Lemma 3.1. There exists a unique operator $C^{l} \in \mathcal{L}((E) \otimes(E),(E))$ such that for any $\xi_{1}, \xi_{2}, \eta_{1} \in E$,

$$
\left\langle\left\langle C^{l}\left(\phi_{\xi_{1}} \otimes \phi_{\xi_{2}}\right), \phi_{\eta_{1}}\right\rangle\right\rangle=e^{\frac{1}{\sqrt{2}}\left\langle\xi_{1}-\xi_{2}, \eta_{1}\right\rangle} .
$$

Proof. For any $p, q \geq 0$, we obtain that

$$
\begin{aligned}
\left|\frac{1}{\sqrt{2}}\langle\xi, \eta\rangle\right| & \leq \frac{1}{\sqrt{2}}|\xi|_{p}|\eta|_{-p} \\
& \leq \frac{1}{\sqrt{2}} \rho^{q}|\xi|_{p+q}|\eta|_{-p} \\
& \leq \frac{1}{8 \epsilon} \rho^{2 q}|\xi|_{p+q}^{2}+\epsilon|\eta|_{-p}^{2}
\end{aligned}
$$

for any $\epsilon>0$. Since for any $\epsilon>0$, there exists $q \geq 0$ such that $\rho^{2 q} \leq 8 \epsilon^{2}$. The right hand side of $(7)$ is denoted by $G\left(\xi_{1}, \xi_{2}, \eta_{1}\right)$. Then by applying (8), we can 
see that $G\left(\xi_{1}, \xi_{2}, \eta_{1}\right)$ satisfies (4) with $m=2$ and $n=1$. Therefore, by Theorem 2.1, there exists a unique operator $C^{l} \in \mathcal{L}((E) \otimes(E),(E))$ such that

$$
\left\langle\left\langle C^{l}\left(\phi_{\xi_{1}} \otimes \phi_{\xi_{2}}\right), \phi_{\eta_{1}}\right\rangle\right\rangle=G\left(\xi_{1}, \xi_{2}, \eta_{1}\right), \quad \xi_{1}, \xi_{2}, \eta_{1} \in E,
$$

which gives the proof.

Lemma 3.2. We have

$$
C^{l}\left(\phi_{\xi_{1}} \otimes \phi_{\xi_{2}}\right)(y)=\left\langle\left\langle\mathcal{R}_{\frac{1}{\sqrt{2}}, \frac{1}{\sqrt{2}} y} \phi_{\xi_{1}}, \mathcal{R}_{\frac{1}{\sqrt{2}},-\frac{1}{\sqrt{2}} y} \phi_{\xi_{2}}\right\rangle\right\rangle, \quad y \in E_{\mathbb{R}}^{*}
$$

for $\xi_{1}, \xi_{2} \in E$.

Proof. For any $\xi_{1}, \xi_{2} \in E$, by applying (5), we see that

$$
\left\langle\left\langle\mathcal{R}_{\frac{1}{\sqrt{2}}, \frac{1}{\sqrt{2}} y} \phi_{\xi_{1}}, \mathcal{R}_{\frac{1}{\sqrt{2}},-\frac{1}{\sqrt{2}} y} \phi_{\xi_{2}}\right\rangle\right\rangle=\phi_{\frac{1}{\sqrt{2}}\left(\xi_{1}-\xi_{2}\right)}(y),
$$

which gives the proof.

From Lemma 3.2, for any $\phi, \psi \in(E)$, we write

$$
\phi *_{\mathrm{Y}}^{l} \psi=C^{l}(\phi \otimes \psi),
$$

which is called the Yeh convolution of $\phi$ and $\psi$ and coincides with the convolution defined in (1).

For each $\alpha, \beta \in \mathbb{C}$, by applying Theorem 2.1 we can easily see that there exists a unique operator $\mathcal{G}_{\alpha, \beta} \in \mathcal{L}((E),(E))$ such that

$$
\widehat{\mathcal{G} \alpha, \beta}(\xi, \eta)=e^{\frac{\alpha}{2}\langle\xi, \xi\rangle+\beta\langle\xi, \eta\rangle}, \quad \xi, \eta \in E .
$$

The operator $\mathcal{G}_{\alpha, \beta}$ is called the Fourier-Gauss transform [2] and its adjoint operator $\mathcal{F}_{\alpha, \beta}=\mathcal{G}_{\alpha, \beta}^{*} \in \mathcal{L}\left((E)^{*},(E)^{*}\right)$ is called the Fourier-Mehler transform. Then we have

$$
\mathcal{G}_{\alpha, \beta}=\Gamma(\beta I) e^{\frac{\alpha}{2} \Delta_{\mathrm{G}}}
$$

and the integral representation:

$$
\mathcal{G}_{\alpha, \beta} \phi(x)=\int_{E_{\mathbb{R}}^{*}} \phi\left(\beta x+\sqrt{\alpha+1-\beta^{2}} y\right) \mu(d y), \quad x \in E_{\mathbb{R}}^{*}
$$

(see $[10,2,5])$. Then the following theorem gives a relation between the Yeh convolution and the Fourier-Gauss transform.

Theorem 3.3. Let $\alpha \in \mathbb{C}$. Then for any $\phi, \psi \in(E)$, we have

$$
\mathcal{G}_{\alpha^{2}, \alpha}\left(\phi *_{\mathrm{Y}}^{l} \psi\right)=\left(\mathcal{G}_{\frac{\alpha^{2}}{2}, \frac{\alpha}{\sqrt{2}}} \phi\right)\left(\mathcal{G}_{\frac{\alpha^{2}}{2},-\frac{\alpha}{\sqrt{2}}} \psi\right),
$$

where the right hand side is the pointwise multiplication.

Proof. For any $\xi, \eta \in E$, by applying (9), we obtain that

$$
\begin{aligned}
\mathcal{G}_{\alpha^{2}, \alpha}\left(\phi_{\xi} *_{\mathrm{Y}}^{l} \phi_{\eta}\right) & =\mathcal{G}_{\alpha^{2}, \alpha} \phi_{\frac{1}{\sqrt{2}}(\xi-\eta)} \\
& =e^{\frac{\alpha^{2}}{4}\langle\xi-\eta, \xi-\eta\rangle} \phi_{\frac{\alpha}{\sqrt{2}}(\xi-\eta)}
\end{aligned}
$$




$$
\begin{aligned}
& =e^{\frac{\alpha^{2}}{4}\langle\xi, \xi\rangle+\frac{\alpha^{2}}{4}\langle\eta, \eta\rangle} \phi_{\frac{\alpha}{\sqrt{2}} \xi} \phi_{-\frac{\alpha}{\sqrt{2}} \eta} \\
& =\left(\mathcal{G}_{\frac{\alpha^{2}}{2}, \frac{\alpha}{\sqrt{2}}} \phi_{\xi}\right)\left(\mathcal{G}_{\frac{\alpha^{2}}{2},-\frac{\alpha}{\sqrt{2}}} \phi_{\eta}\right),
\end{aligned}
$$

which gives the proof of (11).

Theorem 3.4. For any $\phi, \psi, \varphi \in(E)$, we have

$$
\left\langle\left\langle\phi *_{\mathrm{Y}}^{l} \psi, \varphi\right\rangle\right\rangle=\left\langle\left\langle\phi(y),\left\langle\left\langle\psi, \mathcal{R}_{-\frac{1}{\sqrt{2}}, \frac{1}{\sqrt{2}} y} \varphi\right\rangle\right\rangle\right\rangle\right\rangle,
$$

where the right hand side is understood as the bilinear form for the functions of variable $y$.

Proof. For any $\xi_{2}, \eta \in E$, by applying (5), we obtain that

$$
\left\langle\left\langle\phi_{\xi_{2}}, \mathcal{R}_{-\frac{1}{\sqrt{2}}, \frac{1}{\sqrt{2}} y} \phi_{\eta}\right\rangle\right\rangle=e^{-\frac{1}{\sqrt{2}}\left\langle\xi_{2}, \eta\right\rangle} \phi_{\frac{1}{\sqrt{2}} \eta}(y),
$$

which implies that

$$
\begin{aligned}
\left\langle\left\langle\phi_{\xi_{1}}(y),\left\langle\left\langle\phi_{\xi_{2}}, \mathcal{R}_{-\frac{1}{\sqrt{2}}, \frac{1}{\sqrt{2}} y} \phi_{\eta}\right\rangle\right\rangle\right\rangle\right\rangle & =e^{\frac{1}{\sqrt{2}}\left\langle\xi_{1}-\xi_{2}, \eta\right\rangle} \\
& =\left\langle\left\langle\phi_{\xi_{1}} *_{\mathrm{Y}}^{l} \phi_{\xi_{2}}, \phi_{\eta}\right\rangle\right\rangle .
\end{aligned}
$$

Therefore, the proof is immediate from the continuities since exponential vectors span a dense subspace of $(E)$.

From Theorem 3.4, we write

$$
\psi *_{\mathrm{Y}}^{r} \varphi(y)=\left\langle\left\langle\psi, \mathcal{R}_{-\frac{1}{\sqrt{2}}, \frac{1}{\sqrt{2}} y} \varphi\right\rangle\right\rangle, \quad y \in E_{\mathbb{R}}^{*} .
$$

Then we have

$$
\left\langle\left\langle\phi *_{\mathrm{Y}}^{l} \psi, \varphi\right\rangle\right\rangle=\left\langle\left\langle\phi, \psi *_{\mathrm{Y}}^{r} \varphi\right\rangle\right\rangle
$$

for any $\phi, \psi, \varphi \in(E)$.

\section{Convolution of Generalized White Noise Functionals}

In this section, we study an extension of convolution operator to generalized white noise functionals.

Theorem 4.1. There exists an operator $C^{r} \in \mathcal{L}\left((E)^{*} \otimes(E),(E)\right)$ such that

$$
C^{r}(\psi \otimes \varphi)=\psi *_{\mathrm{Y}}^{r} \varphi
$$

for $\psi, \varphi \in(E)$.

Proof. Consider the function $G: E \times E \times E \rightarrow E$ defined by

$$
G\left(\xi_{1}, \eta_{1}, \eta_{2}\right)=e^{\frac{1}{\sqrt{2}}\left\langle\eta_{1}-\eta_{2}, \xi_{1}\right\rangle}, \quad \xi_{1}, \eta_{1}, \eta_{2} \in E .
$$

Then by applying similar arguments used in the proof of Lemma 3.1 and Theorem 2.1, there exists a unique operator $\Xi \in \mathcal{L}((E),(E) \otimes(E))$ such that

$$
\left\langle\left\langle\Xi\left(\phi_{\xi_{1}}\right), \phi_{\eta_{1}} \otimes \phi_{\eta_{2}}\right\rangle\right\rangle=G\left(\xi_{1}, \eta_{1}, \eta_{2}\right), \quad \xi_{1}, \eta_{1}, \eta_{2} \in E .
$$


On the other hand, by the kernel theorem, we have the following topological isomorphisms:

$$
\mathcal{L}\left((E)^{*} \otimes(E),(E)\right) \stackrel{\stackrel{J_{1}}{\rightleftarrows}}{\cong}(E) \otimes(E) \otimes(E)^{*} \stackrel{\stackrel{J_{2}}{\cong}}{\cong} \mathcal{L}((E),(E) \otimes(E)) .
$$

In fact, for any $\Xi \in \mathcal{L}\left((E)^{*} \otimes(E),(E)\right)$ and $\Phi, \Psi \in(E)^{*}, \phi \in(E)$, we obtain that

$$
\begin{aligned}
\langle\langle\Xi(\Phi \otimes \phi), \Psi\rangle\rangle & =\left\langle\left\langle J_{1} \Xi, \Psi \otimes \Phi \otimes \phi\right\rangle\right\rangle \\
& =\left\langle\left\langle\left(J_{2} J_{1} \Xi\right)(\phi), \Psi \otimes \Phi\right\rangle\right\rangle .
\end{aligned}
$$

Put

$$
C^{r}=J_{1}^{-1}\left(J_{2}^{-1}(\Xi)\right)
$$

Then for any $\xi_{1}, \eta_{1}, \eta_{2} \in E$, we obtain that

$$
\begin{aligned}
\left\langle\left\langle C^{r}\left(\phi_{\eta_{2}} \otimes \phi_{\xi_{1}}\right), \phi_{\eta_{1}}\right\rangle\right\rangle & =\left\langle\left\langle J_{1}\left(C^{r}\right), \phi_{\eta_{1}} \otimes \phi_{\eta_{2}} \otimes \phi_{\xi_{1}}\right\rangle\right\rangle \\
& =\left\langle\left\langle J_{2}\left(J_{1}\left(C^{r}\right)\right)\left(\phi_{\xi_{1}}\right), \phi_{\eta_{1}} \otimes \phi_{\eta_{2}}\right\rangle\right\rangle \\
& =\left\langle\left\langle\Xi\left(\phi_{\xi_{1}}\right), \phi_{\eta_{1}} \otimes \phi_{\eta_{2}}\right\rangle\right\rangle \\
& =e^{\frac{1}{\sqrt{2}}\left\langle\eta_{1}-\eta_{2}, \xi_{1}\right\rangle} \\
& =\left\langle\left\langle C^{l}\left(\phi_{\eta_{1}} \otimes \phi_{\eta_{2}}\right), \phi_{\xi_{1}}\right\rangle\right\rangle .
\end{aligned}
$$

Therefore, for any $\xi_{1}, \eta_{1}, \eta_{2} \in E$, we have

$$
\left\langle\left\langle C^{r}\left(\phi_{\eta_{2}} \otimes \phi_{\xi_{1}}\right), \phi_{\eta_{1}}\right\rangle\right\rangle=\left\langle\left\langle\phi_{\eta_{1}}, \phi_{\eta_{2}} *_{\mathrm{Y}}^{r} \phi_{\xi_{1}}\right\rangle\right\rangle=\left\langle\left\langle\phi_{\eta_{2}} *_{\mathrm{Y}}^{r} \phi_{\xi_{1}}, \phi_{\eta_{1}}\right\rangle\right\rangle,
$$

which implies the proof.

For each $\Phi \in(E)^{*}$ and $\phi \in(E)$, from Theorem 4.1, the convolution $\Phi *_{\mathrm{Y}}^{r} \phi \in$ $(E)$ is defined by

$$
\Phi *_{\mathrm{Y}}^{r} \phi=C^{r}(\Phi \otimes \phi) .
$$

Theorem 4.2. The operator $C^{l}$ can be extended to $(E)^{*} \otimes(E)^{*}$ as a continuous linear operator in $\mathcal{L}\left((E)^{*} \otimes(E)^{*},(E)^{*}\right)$ of which the extension is denoted by the same symbol.

Proof. By the dual property, the proof is enough to see that $\left(C^{l}\right)^{*} \in \mathcal{L}((E),(E) \otimes$ $(E))$. For any $\xi_{1}, \eta_{1}, \eta_{2}$, we have

$$
\begin{aligned}
\left\langle\left\langle\left(C^{l}\right)^{*}\left(\phi_{\xi_{1}}\right), \phi_{\eta_{1}} \otimes \phi_{\eta_{2}}\right\rangle\right\rangle & =\left\langle\left\langle C^{l}\left(\phi_{\eta_{1}} \otimes \phi_{\eta_{2}}\right), \phi_{\xi_{1}}\right\rangle\right\rangle \\
& =\left\langle\left\langle\phi_{\eta_{1}} *_{\mathrm{Y}}^{l} \phi_{\eta_{2}}, \phi_{\xi_{1}}\right\rangle\right\rangle \\
& =e^{\frac{1}{\sqrt{2}}\left\langle\eta_{1}-\eta_{2}, \xi_{1}\right\rangle} .
\end{aligned}
$$

Then by applying similar arguments used in the proof of Lemma 3.1, we see that $\left(C^{l}\right)^{*} \in \mathcal{L}((E),(E) \otimes(E))$. Therefore, $C^{l} \in \mathcal{L}\left((E)^{*} \otimes(E)^{*},(E)^{*}\right)$.

For each $\Phi, \Psi \in(E)^{*}$, the Yeh convolution $\Phi *_{\mathrm{Y}}^{l} \Psi \in(E)^{*}$ is defined by

$$
\Phi *_{Y}^{l} \Psi=C^{l}(\Phi \otimes \Psi) .
$$


Theorem 4.3. For any $\Phi, \Psi \in(E)^{*}$ and $\varphi \in(E)$, we have

$$
\left\langle\left\langle\Phi *_{\mathrm{Y}}^{l} \Psi, \varphi\right\rangle\right\rangle=\left\langle\left\langle\Phi, \Psi *_{\mathrm{Y}}^{r} \varphi\right\rangle\right\rangle .
$$

Proof. The proof is immediate by the definitions of the convolutions $*_{\mathrm{Y}}^{l}$ and $*_{\mathrm{Y}}^{r}$.

The following theorem gives a relation between the Yeh convolution and the Fourier-Mehler transform.

Theorem 4.4. Let $\alpha \in \mathbb{C}$. Then for any $\Phi, \Psi \in(E)^{*}$, we have

$$
\left(\mathcal{F}_{\alpha, 1} \Phi\right) *_{\mathrm{Y}}^{l}\left(\mathcal{F}_{\alpha, 1} \Psi\right)=\mathcal{F}_{\alpha, 1}\left(\Phi *_{\mathrm{Y}}^{l} \Psi\right) \text {. }
$$

Proof. Note that for any $\alpha \in \mathbb{C}, \beta \in \mathbb{C} \backslash\{0\}$ and $\xi \in E$,

$$
\Gamma(\beta I) e^{\alpha \Delta_{\mathrm{G}}} \phi_{\xi}=e^{\alpha\langle\xi, \xi\rangle} \phi_{\beta \xi}=e^{\alpha \beta^{-2} \Delta_{\mathrm{G}}} \Gamma(\beta I) \phi_{\xi},
$$

which implies that

$$
\Gamma(\beta I) e^{\alpha \Delta_{\mathrm{G}}}=e^{\alpha \beta^{-2} \Delta_{\mathrm{G}}} \Gamma(\beta I) .
$$

Also, we note that for any $\alpha \in \mathbb{C}$ and $y \in E^{*}, e^{a(y)} e^{\alpha \Delta_{\mathrm{G}}}=e^{\alpha \Delta_{\mathrm{G}}} e^{a(y)}$. Therefore, by (6) and (10), we obtain that

$$
\begin{aligned}
\Psi *_{\mathrm{Y}}^{r} \mathcal{G}_{\alpha, 1} \phi & =\left\langle\left\langle\Psi, \Gamma\left(-\frac{1}{\sqrt{2}} I\right) e^{-\frac{1}{4} \Delta_{\mathrm{G}}} e^{\frac{1}{\sqrt{2}} a(y)} e^{\frac{\alpha}{2} \Delta_{\mathrm{G}}} \phi\right\rangle\right\rangle \\
& =\left\langle\left\langle\Psi, e^{\alpha \Delta_{\mathrm{G}}} \Gamma\left(-\frac{1}{\sqrt{2}} I\right) e^{-\frac{1}{4} \Delta_{\mathrm{G}}} e^{\frac{1}{\sqrt{2}} a(y)} \phi\right\rangle\right\rangle \\
& =\left(\mathcal{F}_{2 \alpha, 1} \Psi\right) *_{\mathrm{Y}}^{r} \phi,
\end{aligned}
$$

and

$$
\mathcal{G}_{\alpha, 1}\left(\phi_{\xi} *_{\mathrm{Y}}^{r} \phi_{\eta}\right)=e^{-\frac{1}{\sqrt{2}}\langle\xi, \eta\rangle+\frac{\alpha}{4}\langle\eta, \eta\rangle} \phi_{\frac{1}{\sqrt{2}} \eta}=\phi_{\xi} *_{\mathrm{Y}}^{r}\left(\mathcal{G}_{\frac{\alpha}{2}, 1} \phi_{\eta}\right),
$$

which implies that

$$
\mathcal{G}_{\alpha, 1}\left(\Psi *_{\mathrm{Y}}^{r} \varphi\right)=\Psi *_{\mathrm{Y}}^{r}\left(\mathcal{G}_{\frac{\alpha}{2}, 1} \varphi\right), \quad \Psi \in(E)^{*}, \quad \varphi \in(E) .
$$

Therefore, we obtain that

$$
\begin{aligned}
\left\langle\left\langle\mathcal{F}_{\alpha, 1} \Phi *_{\mathrm{Y}}^{l} \mathcal{F}_{\alpha, 1} \Psi, \varphi\right\rangle\right\rangle & =\left\langle\left\langle\Phi, \mathcal{G}_{\alpha, 1}\left(\mathcal{F}_{\alpha, 1} \Psi *_{\mathrm{Y}}^{r} \varphi\right)\right\rangle\right\rangle \\
& =\left\langle\left\langle\Phi,\left(\Psi *_{\mathrm{Y}}^{r} \mathcal{G}_{\frac{\alpha}{2}, 1} \mathcal{G}_{\frac{\alpha}{2}, 1} \varphi\right)\right\rangle\right\rangle \\
& =\left\langle\left\langle\Phi,\left(\Psi *_{\mathrm{Y}}^{r} \mathcal{G}_{\alpha, 1} \varphi\right)\right\rangle\right\rangle \\
& =\left\langle\left\langle\mathcal{F}_{\alpha, 1}\left(\Phi *_{\mathrm{Y}}^{l} \Psi\right), \varphi\right\rangle\right\rangle,
\end{aligned}
$$

which gives the proof of (12). 


\section{REFERENCES}

1. D.M. Chung, T.S. Chung and U.C. Ji, A simple proof of analytic characterization theorem for operator symbols, Bull. Korean Math. Soc. 34 (1997), 421-436.

2. D.M. Chung and U.C. Ji, Transforms groups on white noise functionals and their applications, Appl. Math. Optim. 37 (1998), 205-223.

3. L. Gross, Potential theory on Hilbert space, J. Funct. Anal. 1 (1967), 123-181.

4. T. Hida, Analysis of Brownian Functionals, Carleton Math. Lect. Notes 3, 241-272.

5. M.K. Im, U.C. Ji and Y.J. Park, Relations between the first variation, the convolutions and the generalized Fourier-Gauss transforms, Bull. Korean Math. Soc. 48 (2011), 291-302.

6. U.C. Ji and Y.Y. Kim, Convolution of white noise operators, Bull. Korean Math. Soc. 48 (2011), 1003-1014.

7. U.C. Ji and N. Obata, A unified characterization theorem in white noise theory, Infin. Dim. Anal. Quantum Probab. Related Topics 6 (2003), 167-178.

8. H.-H. Kuo, White Noise Distribution Theory, CRC Press, 1996.

9. H.-H. Kuo, J. Potthoff and L. Streit, A characterization of white noise test functionals, Nagoya Math. J. 121 (1991), 185-194.

10. Y.J. Lee, Integral transforms of analytic functions on abstract Wiener spaces, J. Funct. Anal. 47 (1982), 153-164.

11. N. Obata, An analytic characterization of symbols of operators on white noise functionals, J. Math. Soc. Japan 45 (1993), 421-445.

12. N. Obata, White Noise Calculus and Fock Space, Lect. Notes on Math. 1577, SpringerVerlag, Berlin, 1994.

13. J. Potthoff and L. Streit, A characterization of Hida distributions, J. Funct. Anal. 101 (1991), 212-229.

14. J. Yeh, Convolution in Fourier-Wiener transform, Pacific J. Math. 15 (1965), 731-738.

Un Cig Ji received M.Sc. in 1991 and Ph.D in 1996 from Sogang University. He is currently a professor at Chungbuk National University since 2001. His research interests are Functional Analysis, Infinite Dimensional Analysis, White Noise Theory and Quantum probability.

Department of Mathematics, Research institute of Mathematical Finance, Chungbuk National University, Cheongju 361-763, Korea.

e-mail: uncigji@chungbuk.ac.kr

Young Yi Kim received M.Sc. and Ph.D. from Chunbuk National University. Her research interests are Functional Analysis and White Noise Theory.

Research institute for Natural Science, Hanyang University, Seoul 133-791, Korea.

e-mail: kimyy@chungbuk.ac.kr

Yoon Jung Park received M.Sc. from Chunbuk National University. Her research interests are White Noise Theory.

Department of Mathematics, Chungbuk National University, Cheongju 361-763, Korea.

e-mail: yjpark@chungbuk.ac.kr 